\title{
Streptococcus bovis Endocarditis after Colonic Polypectomy
}

\author{
Napohn Chongprasertpon ${ }^{1}$, Ronan Cusack ${ }^{1}$, J J Coughlan ${ }^{1}$, Wing Tung Chung ${ }^{2}$, Chun Ho Leung ${ }^{2}$, Thomas J Kiernan ${ }^{1}$ \\ ${ }^{1}$ Department of Cardiology, University Hospital Limerick, Dooradoyle, Limerick, Ireland \\ ${ }^{2}$ Faculty of Medicine, The Chinese University of Hong Kong, Ma Liu Shui, Hong Kong SAR, The People's Republic of China
}

\section{Doi: 10.12890/2019_001110 - European Journal of Case Reports in Internal Medicine - @ EFIM 2019}

Received: 01/04/2019

Accepted: 02/04/2019

Published: 08/05/2019

How to cite this article: Chongprasertpon N, Cusack R, Coughlan JJ, Chung WT, Leung CH, Kiernan TJ. Streptococcus bovis endocarditis after colonic polypectomy. EJCRIM 2019;6: doi:10.12890/2019_001110.

Conflicts of Interests: The Authors declare that there are no competing interests.

Acknowledgements: We acknowledge the Cardiac Diagnostics Department in University Hospital Limerick for their high-quality images. We also acknowledge all other healthcare professionals who have provided care for the patient.

This article is licensed under a Commons Attribution Non-Commercial 4.0 License

\section{ABSTRACT}

We describe a case of Streptococcus lutetiensis infective endocarditis occurring in a patient following colonic polypectomy. The patient had multiple risk factors for infective endocarditis including pre-existing mitral valve prolapse and regurgitation. Transoesophageal echocardiography revealed a friable mass on the posterior mitral valve leaflet, confirming the diagnosis. The patient was treated with intravenous antibiotics, successfully underwent mitral valve surgery and was discharged home for outpatient follow-up.

This report details an uncommon case presentation, highlights areas for improvement in clinical practice, and summarises the current knowledge available in the literature regarding Streptococcus bovis infective endocarditis.

\section{LEARNING POINTS}

- Infective endocarditis occurring in association with gastrointestinal endoscopy is rare.

- Clinical suspicion of infective endocarditis after colonic polypectomy or biopsy should be maintained, especially in those with risk factors for infective endocarditis.

- Antibiotic prophylaxis against infective endocarditis is not recommended for routine gastrointestinal endoscopic procedures.

\section{KEYWORDS}

Infective endocarditis, colonic polypectomy, Streptococcus bovis

\section{CASE PRESENTATION}

A 71 year old man was brought to the hospital by ambulance following a witnessed syncopal episode. Of note, 3 weeks earlier, our patient had undergone colonoscopy with polypectomy of three polyps. Histological analysis of these lesions revealed low-grade dysplasia. He had no other significant background history and was not taking any medications on admission. Examination revealed a pansystolic murmur, loudest at the left fifth intercostal space, mid-clavicular line. The rest of the clinical examination was normal. Renal function was normal on biochemistry, however troponins and C-reactive protein (CRP) were elevated at $28 \mathrm{ng} / \mathrm{l}$ (normal: 0-14 ng/l) and 72 mg/l (normal: 0-5 $\mathrm{mg} / \mathrm{l})$, respectively. CT of the brain, Holter monitoring and carotid Dopplers showed no abnormalities apart from minor atheroma on the external carotid arteries bilaterally. The transthoracic echocardiograph (TTE) displayed mitral valve prolapse with thickening of the mitral valve leaflets and severe mitral regurgitation suspicious for infective endocarditis (IE) (Fig. 1). The patient had undergone a previous TTE 1 year earlier which had shown thickened appearing mitral valve leaflets with prolapse of the posterior mitral valve (P1/P2 scallops) and moderate anteriorly directed mitral regurgitation.

One day after admission the patient developed pyrexia of $38^{\circ} \mathrm{C}$ and a full septic screen was performed including blood cultures. These were subsequently positive for Streptococcus lutetiensis in both the aerobic and anaerobic bottles after 23 and 16 hours of incubation, respectively. 
This was sensitive to penicillin and high-level gentamicin resistance was absent, so gentamicin could be used for synergy.

Advice was sought from microbiology. In view of the association of S. lutetiensis with IE as well as with colonic carcinoma, the polyp histopathology was reviewed. This confirmed adenomas of low-grade dysplasia with no evidence of invasion. We proceeded to transoesophageal echocardiography (TOE) which revealed a large mobile friable mass on the posterior mitral valve leaflet suspicious for IE (Fig. 2).

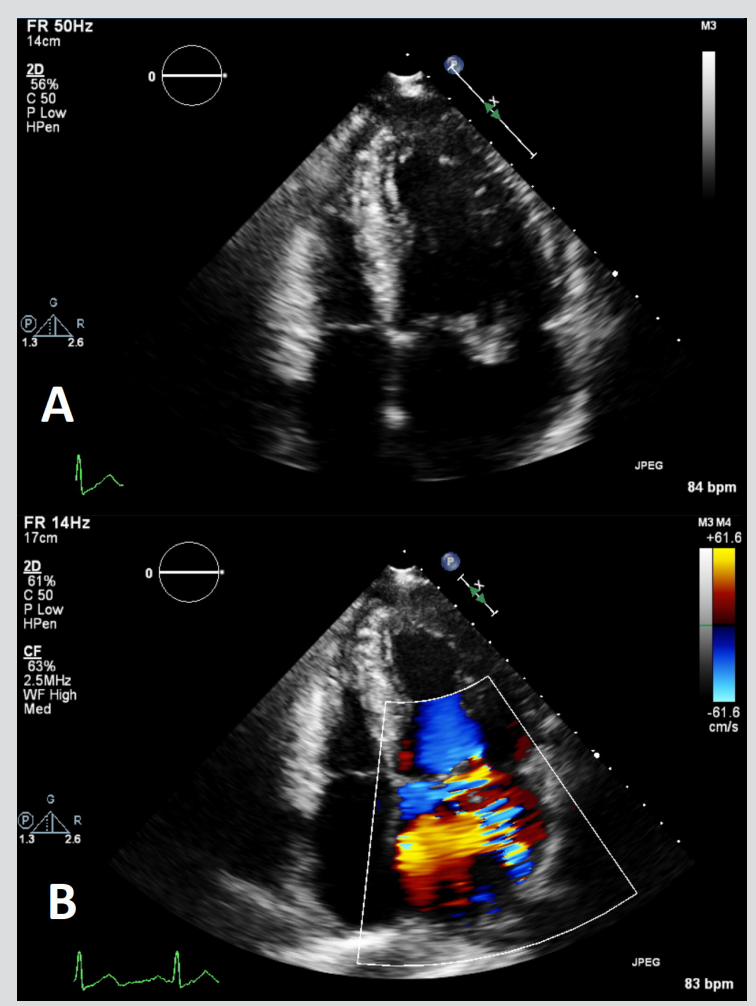

Figure 1. TTE images showing mitral valve prolapse with thickening of the mitral valve leaflets suspicious for infective endocarditis (A) and severe mitral regurgitation seen on colour flow imaging $(B)$

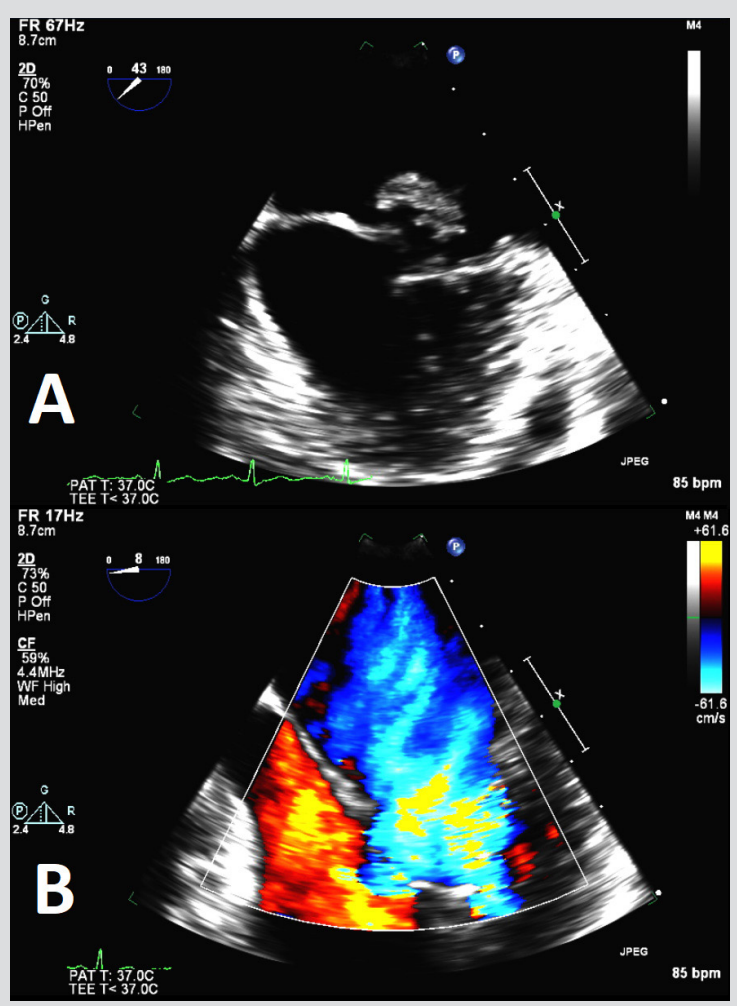

Figure 2. TOE images showing a large mobile friable mass on the posterior mitral valve leaflet suspicious for infective endocarditis (A). Mitral regurgitation could be seen on the colour flow image (B)

The patient was diagnosed with IE after fulfilling one major and three minor modified Duke criteria. The major criterion fulfilled was the echocardiogram findings positive for IE, while the minor criteria were the patient's multiple temperature spikes $\geq 38^{\circ} \mathrm{C}$, the predisposing mitral valve prolapse with regurgitation and the positive blood cultures which did not meet the major criteria. The most likely source was gastrointestinal due to the patient's recent polypectomy. The patient was treated with benzylpenicillin and gentamicin while awaiting transfer to a cardiothoracic centre for surgical intervention.

The patient underwent cardiac surgery during which the anterior and posterior leaflets were found to be abnormal, myxomatous and thickened with large vegetations on both. The valve was excised and a mitral biological prosthesis was placed. Washings were taken from the valves and sent for culture along with the excised valvular tissue; in both cases microbiology results were negative after extended culture. Eleven days post-operatively the patient was transferred back to our cardiology department having been commenced on warfarin with a proposed duration of 3 months, while the antibiotic therapy was switched to vancomycin and meropenem. The patient completed a 6-week course of intravenous antibiotics without issue and is being followed up in our outpatient clinic.

\section{DISCUSSION}

Non-enterococcal Group D streptococci were reportedly found in the intestinal flora in 55\% of patients with colonic carcinoma and $19 \%$ of those with non-colonic neoplasms ${ }^{[1]}$. The association of Streptococcus bovis endocarditis and bacteraemia with colonic neoplasms, polyps, adenocarcinoma and carcinoma has been well documented ${ }^{[2]}$. The European of Society of Cardiology (ESC) guidelines recommend that colonoscopy should be performed during hospitalisation for S. bovis or Streptococcus gallolyticus IE and if negative, annual colonoscopy is suggested $^{[3]}$. 
The S. lutetiensis subspecies is a rare cause of IE. Previous research reported only one case of S. lutetiensis following subspecies analysis of cardiac valve and blood cultures in $17 \mathrm{~S}$. bovis endocarditis patients ${ }^{[4]}$. S. lutetiensis is a member of the S. bovis group and was previously known as $\mathrm{S}$. bovis biotype II/1 prior to the change in taxonomic nomenclature ${ }^{[1]}$.

The valve culture for this patient was negative. However, this is not unusual, particularly following antibiotic therapy. Previous research found only $39.4 \%$ of IE patients had positive valve cultures and $100 \%$ of those with negative valve cultures were receiving antimicrobial therapy ${ }^{[5]}$. The diagnostic value of performing valve cultures has been questioned due to the poor sensitivity and positive predictive value ${ }^{[5]}$. The propensity of $S$. bovis associated with colonic neoplasms to progress to bacteraemia and ultimately endocarditis has been an area of ongoing study. Studies of the genomic sequence of $S$. gallolyticus uncovered an ability to synthesise a number of polysaccharides for the assembly of capsular sheath, collagen-binding proteins and three types of pili; this may account for the ability of this organism to bind to tissues, and thus afford it a particular potency for causing bacteraemia and endocarditis ${ }^{[6]}$.

With regard to antibiotic prophylaxis for gastrointestinal endoscopy, it is estimated that over 14 million colonoscopies are performed in the United States on an annual basis but only approximately 25 cases of IE have been reported in association with endoscopy[7]. The rates of transient bacteraemia which occurs from daily activities such as tooth brushing and flossing (20-68\%), often exceeds those associated with endoscopic procedures ${ }^{[7]}$. The 2015 ESC guidelines for IE recommend prophylaxis consideration only in high-risk patients and only for high-risk procedures such as dental procedures with oral mucosa perforation or requiring manipulation of the gingival or periapical region of the teeth ${ }^{[3]}$. Specifically, antibiotics are not recommended for routine respiratory or gastrointestinal endoscopic procedures ${ }^{[3]}$. High-risk patients include those with prior IE, prosthetic valves or pre-existing cyanotic congenital heart disease (CHD) or any CHD repaired with prosthetic material up to 6 months after the procedure or lifelong if valvular regurgitation or residual shunt remains; those with pre-existing uncomplicated valvular pathology such as mitral valve prolapse/regurgitation like the patient in our case are not considered high risk for this recommendation ${ }^{[3]}$.

\section{REFERENCES}

1. Tripodi MF, Fortunato R, Utili R, Triassi M, Zarrilli R. Molecular epidemiology of Streptococcus bovis causing endocarditis and bacteraemia in Italian patients. Clin Microbiol Infect 2005;11(10):814-819.

2. Zarkin BA, Lillemoe KD, Cameron JL, Effron PN, Magnuson TH, Pitt HA. The triad of Streptococcus bovis bacteremia, colonic pathology, and liver disease. Ann Surg 1990;211:786-791.

3. Habib G, Lancellotti P, Antunes MJ, Bongiorni MG, Casalta JP, Del Zotti F, et al. 2015 ESC Guidelines for the management of infective endocarditis: The Task Force for the Management of Infective Endocarditis of the European Society of Cardiology (ESC). Endorsed by: European Association for Cardio-Thoracic Surgery (EACTS), the European Association of Nuclear Medicine (EANM). Eur Heart J 2015;36(44):3075-3128.

4. Alozie A, Köller K, Pose L, Raftis M, Steinhoff G, Westphal B, et al. Streptococcus bovis infectious endocarditis and occult gastrointestinal neoplasia: experience with 25 consecutive patients treated surgically. Gut Pathog 2015;7:27.

5. Muñoz P, Bouza E, Marín M, Alcalá L, Rodríguez Créixems M, Valerio M, et al. Heart valves should not be routinely cultured. J Clin Microbiol $2008 ; 46(9): 2897-2901$.

6. Rusniok C, Couvé E, Da Cunha V, El Gana R, Zidane N, Bouchier C, et al. Genome sequence of Streptococcus gallolyticus: insights into its adaptation to the bovine rumen and its ability to cause endocarditis. J Bacteriol 2010;192(8):2266-2276.

7. ASGE Standards of Practice Committee, Khashab MA, Chithadi KV, Acosta RD, Bruining DH, Chandrasekhara V, et al. Antibiotic prophylaxis for GI endoscopy. Gastrointest Endosc 2015;81(1):81-89. 